\title{
Enriched Integrations of ERP and PLM in the loT World
}

\author{
Ritesh Jain \\ Senior Software Development Consultant \\ Pune India
}

\begin{abstract}
While the software systems like Product Lifecycle Management (PLM) and Enterprise Resource Planning (ERP) have been solving critical problems of the industries, there are various integrations implemented to use them under overlapping conditions depending on the category of sector the solutions are implemented into. With the new buzzword called Internet of Things, in the Information Technology fraternity the scope and face of various traditional software systems is changing fast. Internet of Things is growing big day-by-day and finding its applications in domestic as well as industrial areas and even to some very unconventional areas as well. This paper discusses the overlapping roles of PLM and ERP systems in the IoT arena. The applications of both the systems can be enhanced and put to better use with the usage of IoT along with.
\end{abstract}

With some of the enhancements, especially in the integration systems implemented for them, helps increasing the productivity and efficiency of the products and also save the cost with early indications of any failure.

\section{General Terms}

Internet of things, Product Lifecycle Management, Enterprise Resource Planning

\section{Keywords}

Internet of Things (IoT), Product Lifecycle Management(PLM), Enterprise Resource Planning (ERP)

\section{INTRODUCTION}

The software products related to managing different (not necessarily separate) components of the industry have long been organized under different categories e.g. (Enterprise Resource Planning) ERP, Customer Relationship Management (CRM), Supply Chain Management (SCM), Product Lifecycle Management (PLM) systems etc. They primarily cater to different functions in an industry and in some of the cases adopted by a particular type of industry e.g. the CRM have been used by an industry that needed more customer interaction on a frequent basis e.g. Telecom companies or Banks. The ERP is primarily used by the companies having a vast range of functions corresponding to the various modules of the ERP system viz. HR, Sales, Finance, and Inventory Management, Purchase etc. So it's used by companies engaged into manufacturing of some kind of product and selling them as well. With the variety of functions in the company there was a risk of them operating in silos and hence was the need of an ERP system to have them working in sync and alleviate the losses and inefficiencies causes by that. The PLM software on the other hand was evolved when there was a need to manage design data of a product development company. The design data consists of mainly the Computer Aided Design (CAD) and associated or non-associated but relevant data. When the volume of CAD data increased beyond human-manageable capacity, PLM software came into the game.

With the advent of usage of these software systems and the increased complexity of integrating various software systems, the customer demanded overlapping features in all of them to avoid the issues of using (read buying) different software systems for a small need of some part of the complementary software.

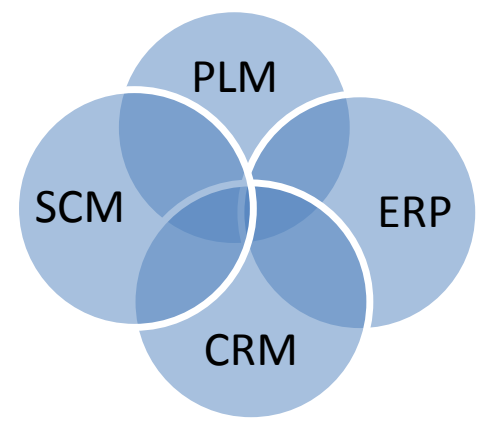

Figure 1: Overlapping roles of different systems

\section{ERP SYSTEMS: WHAT GOOD ARE THEY FOR?}

Information is the key to success of any organization. Precise information at the right time with a click of button has been a fancy of the think-tanks across the industries. Most of the time it's the right decisions at the right time that can help an industry or an initiative of the industry, to succeed. And right decisions in turn attribute their success to the information available at that time. The holistic approach that has been missing in the pre-ERP era has been the major hurdle in taking right decisions. A simple example could be for instance if an industry involved in manufacturing an engineering product that needed some raw material to be available. The low level of inventory when it's needed in the production cycle causes a delay in production that in turn can lead to losses while a more-than-needed level of inventory increases the inventory carrying cost(ICC) causing the increased input cost. This principle has been taught as one of the basics in the relevant branches of engineering as well as management but knowing the level of inventory in a real-time manner has been a challenge if that was done as part of some protocol-based manual process. In the same industry if the sales guy receives an order of a specified quantity at a particular time, he need to know based on the production levels, (that in turn depend on various factors including the inventory level of the raw material) if it's really possible to supply the order within the committed schedule. ERP tried bridging the gaping gaps of such situations. With a properly customized and implemented ERP system, it has become possible to get the information at the right time and most importantly in most 
usable/consumable form related to the various functions in the industry.

Enterprise resource planning (ERP) systems are powerful software packages that enable businesses to integrate a variety of disparate functions. In particular, ERP systems can provide the foundation for a wide range of e-commerce based processes, including web-based ordering and order tracing, inventory management, and built-to-order goods. An ERP controls all aspects of manufacturing including inventory, purchase, process planning, production scheduling, warehouse, delivery, human resources, finance, configurations, effectivity status etc.

\section{PLM SYSTEMS: WHAT GOOD ARE THEY FOR?}

Because of its potential benefits to shorten innovation leadtimes and to reduce costs, PLM has attracted a lot of attention at industry and at research. Product Lifecycle Management as the name suggests, provides a data warehouse to the companies who would like their product data to be managed. It provides hosts of different services e.g.

- Allows capturing the various stages in the product development right from conceptualization, design, design reviews, production planning, generation of drawings and Bill of material, manufacturing data including the details relevant to manufacturing, machining and servicing of the product.

- $\quad$ Provide the CRUD (Create, Retrieve, Update and Delete) services like any other Database system to manage the design data.

- Share the data to allow working in decentralized and distributed mode of work environment and even work with vendors and suppliers.

PLM not only helps one single company but a globally distributed, interdisciplinary collaboration between producers, suppliers, partners and customer. PLM systems, nowadays, also don the avatar of a Cloud-based solution. They can integrate with an ERP system and so on. The PLM systems in many cases need customizations to suit to the different kind of business needs, process needs of the customer etc.

\subsubsection{Evolution of PLM}

With the increase in adoption of CAD data in the industry and increase in spreading footprint of CAD to encompass various type of industries e.g. Furniture, Apparel, Shipping apart from being used in Manufacturing, Engineering, Automotive and Aerospace industry and also with the increase of size of data in CAD systems, there was a need to manage the data using some intelligent and automated system and this gave birth to the PLM system.

As gathered, (Shilovitsky 2013) and (Mahadik 2013) the initial systems to manage the CAD data were primarily the tailor-made/custom-made solutions that were called Product Data Management (PDM). They were developed in-house or bought from vendors that implemented it with a focused approach of solving the particular problem of the keeping and managing the $\mathrm{CAD}$ data. The projects that were developed custom made were gradually converted to solutions and then to generic products. With time it was realized to have a holistic system to manage not just the product data but the whole gamut of activities revolved around them. That's when the term PLM was coined and conceptualized. So the PLM evolved from the nineties' product data management(PDM) approach. A typical PLM system, as mentioned earlier facilitates all CRUD operations and provides the following features, Out-of-the-box(OOTB) (Mule 2012):

- Data Management/Document Management

- Part/Product data Management/Configuration Management

- Process Management/ Workflow Management

- Program Management/ Project Management

- Collaboration Management

- Visualization of data

- Integration with other systems

- Infrastructure Management

- Product Review

- $\quad$ Change management

\section{PLM INTEGRATIONS WITH ERP}

The above mentioned features primarily help in the integration of the CAD with PLM but as the need arise, there were integrations implemented and offered between PLMERP and PLM-CRM also.

The PLM manages the virtual form of the product and ERP's role encapsulates manufacturing the product and delivering it (Mckinney 2015). There are some overlapping functions like managing the part identification, scheduling, process or workflow management, product structure etc.

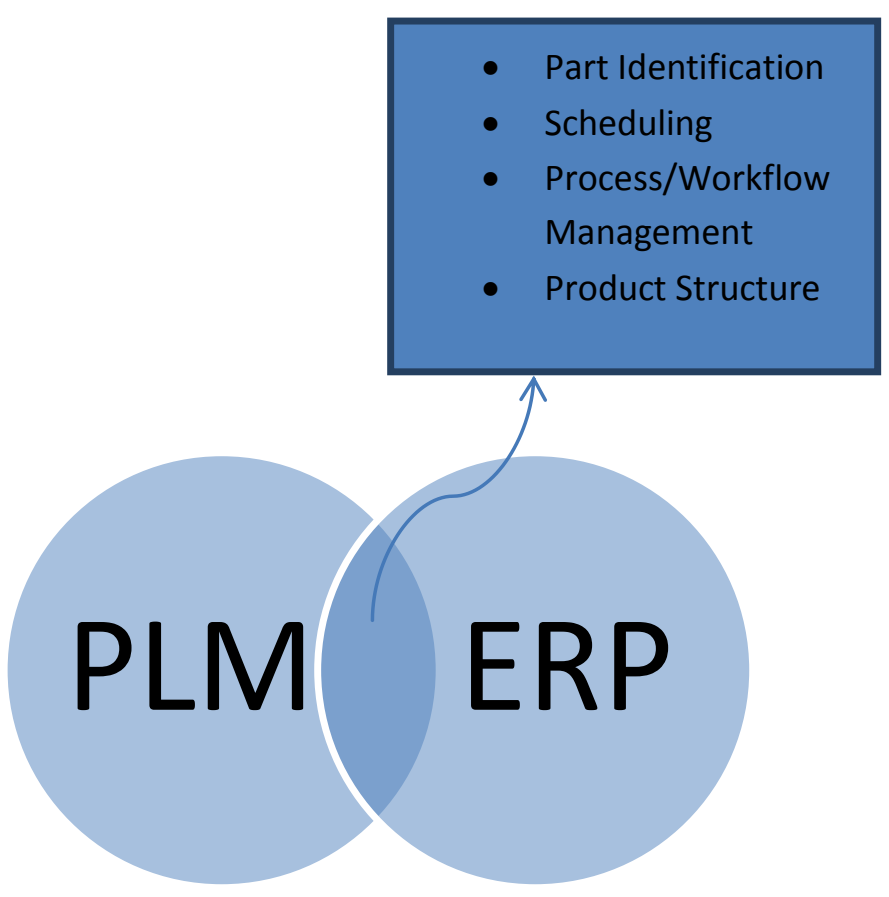

Figure 2: Intersection points of PLM and ERM

For such product data integration, engineering bill of materials (EBOM) needs to be transformed to manufacturing bill of materials (MBOM), but the MBOM transformation 
must be done in such a way as to fit the particularities of each manufacturing site.

Both PLM and ERP have their distinct advantages and hence they are needed to cater to specific purposes in an industry. Since they have some overlapping functions it's important to implement the integrations between them, properly so that the data exchange between is smooth and synchronized. This becomes further challenging because of the different vendors supplying these systems. These software products are so big that no one company can develop these products.

\section{AN ERP SOLUTION WITHOUT PLM}

Business relying only on ERP without a PLM system finds it difficult to manage the product data. The resulting scrap and rework around the product in the office and the shop floor is often costly. By implementing a PLM system beforehand, an organization can be confident the product information is being accurately managed and the ERP system is working with correct data.

Because ERP systems intend to read the BOM for transactional purposes only, they do not have the product data collaboration-ready among the departments. Preserving the history of change information is important to understand the change and the time it occurred. Without a proper PLM system in place there is a risk of losing product change information that helps accurate financial planning. By keeping product data in a PLM system and then exchanging it with an ERP system, the product company enjoys a more synchronized, cohesive and efficient manufacturing process.

Global manufacturing companies must implement manufacturing engineering with proper consideration given to the manufacturing process and resources that are appropriate for the particularities of each manufacturing site. Particularly for effective enterprise resource planning (ERP) system implementation under a global manufacturing environment, the PDM or PLM integration for product data is one of the important keys to success.

To have an ERP and PLM system working in tandem with each other, they need to be integrated properly with the same conventions followed in both of them. The overlapping areas need to be taken care of, more carefully to have smooth exchange of information between the two. Consider a scenario of an assembly design that is saved in a PLM system along with its supporting documents that include some nonmechanical components e.g. lubricating oil. The quantity of oil, specified in the PLM system, has to be passed to the ERP system that can track the work-order, capture the procurement details and manage the inventory of the same. Both the PLM and ERP systems have to follow the similar conventions to ensure smooth flow of information across and make the overall implementation successful.

\section{WHAT'S DIFFERENT WITH IOT}

Internet of Things is fast finding the users both on industrial as well as domestic front. As the Wikipedia says: "The
Internet of things (IoT) is the inter-networking of physical devices, vehicles (also referred to as "connected devices" and "smart devices"), buildings, and other items - embedded with electronics, software, sensors, actuators, and network connectivity that enable these objects to collect and exchange data."

A popular magazine says "The Internet of Things will be the largest device market in the world. By an estimate, by 2019 it will be more than double the size of the smartphone, PC, tablet, connected car, and the wearable market combined." (GREENOUGH n.d.). Gartner, Inc. forecasts that connected things through IOT will reach 20.8 billion by 2020. (Gartner n.d.)

So it is obvious for the industry to have the focus on IoT. Internet of things has wide range of applications and as listed by IOT Analytics the below mentioned are the top ten out of them. (Lueth 2015)

1. Smart home

2. Wearables

3. Smart City

4. Smart grids

5. Industrial internet

6. Connected car

7. Connected Health (Digital health/Telehealth/Telemedicine)

8. Smart retail

9. Smart supply chain

10. Smart farming

But out of these as the Gartner suggests, the most commercial application is the industrial one. In fact, it has been given a specific name i.e. Industrial Internet of Things (IIoT). In industries there are applications ranging from catching the leakage in pipes in a process plant to tracking or anticipating the breakdown of a manufacturing center in a manufacturing company. The biggest advantage is to have the capability to collect, manage and control the information and devices remotely.

PLM can accommodate and process the data coming from the sensors attached to the live product in the field. This live information can be fed to be processed for various purposes e.g. it can be used to amend the future design to better the product by considering the usage of the product and its functional behavior under the actual operating conditions or the overload conditions. It can be used to monitor or track the current status of the product or process and enable taking the appropriate actions. In fact, there are advance IIoT systems implemented that can take the actions automatically. 


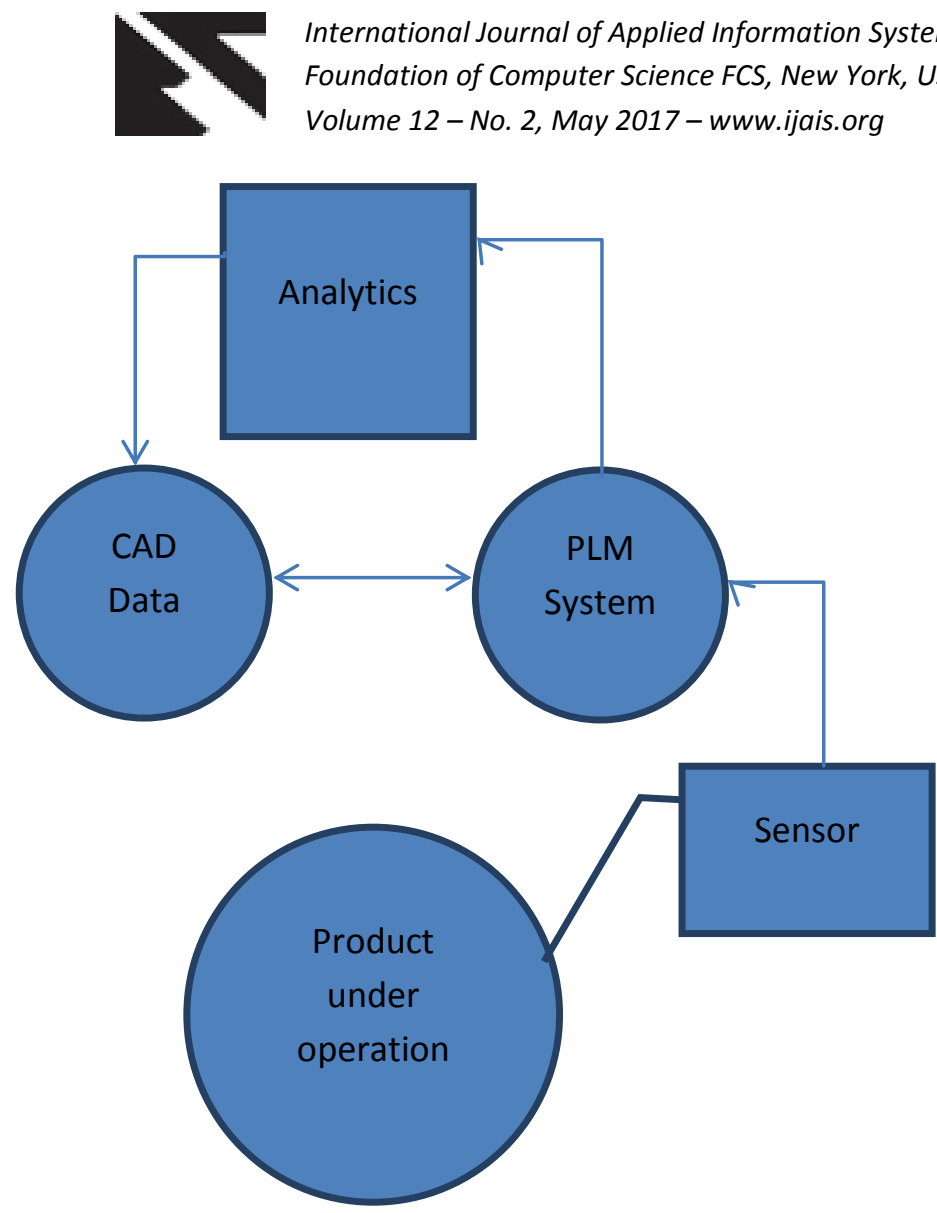

Figure 3 : IOT with PLM System

In the IOT arena, the applications can be different with ERP and PLM. The integration of PLM system with IOT could be useful at the $\mathrm{CAD}$ vendor end to enable it improving the design and application of the product based on the feedback information fed to it but the ERP system implemented at the customer site would enable improving the availability of the product using the supplied/designed product that have sensors attached to it.

\section{USE-CASE SCENARIOS WITH IOT}

1. With the IOT, one of the use cases is to analyze the breakdown frequency of the product or predicting the breakdown of the machine using the sensor attached product and allow preparing for the breakdown maintenance of the machine. This would also enable the customer to maintain the inventory of the spares used for the product in the ERP system.

2. It can also be used to predict the load on the machine to allow planning for the preventive maintenance of the machine, or prepare schedule of the preventive maintenance of the machine and ensure supply of the spares. This can also provide inputs to the Human Resources department of the company to plan resource allocation to the respective departments e.g. in production, maintenance, purchase etc. It can also help hiring contract employees based on the prediction of demand.

3. The sensor data can also give early indications of any delivery slippages in the supply chain module of ERP, due to the breakdowns.

4. The breakdown or fault analysis of the sensor data of a product received from field can be used to
- Understand the conditions causing the frequent failure of the product.

- Identify the lot that used some of the components from a particular vendor that fails frequently.

- Identify the lot manufactured on a particular machine.

- Identify the lot manufactured by a particular machinist.

- Identify the lot manufactured under some different conditions of the plant.

The PLM receives the data from the sensors incorporated in the product in the field and the data could aid in deciding of replacing a particular component with another one available in the ERP system and can help doing the feasibility study of the same.

Additionally, the ERP system can capture the feedback from the customer and help modifying the design so as to make it more robust and long lasting. This can also be based on the frequency of service request of the product.

5. The sensors incorporated on a product operating in field can send data to the IIOT (Industrial Internet of Things) Server. It could be early signals of a breakdown or excessive vibrations coming out of one of the spares. The IIOT server will in turn collect the information including the intensity and frequency of the problem.

If the problem is recurring and severe the IOT could send the request to the PLM to get an alternate spare available from the same or another vendor. The information related to the spare could be fetched from the ERP system e.g. the rating of the vendor, expected delivery time, price comparisons by involving the purchase module of the ERP system. These iterations could help deciding the best alternate spare available and could be fed to the CAD system to do analyses like FEA or simulation.

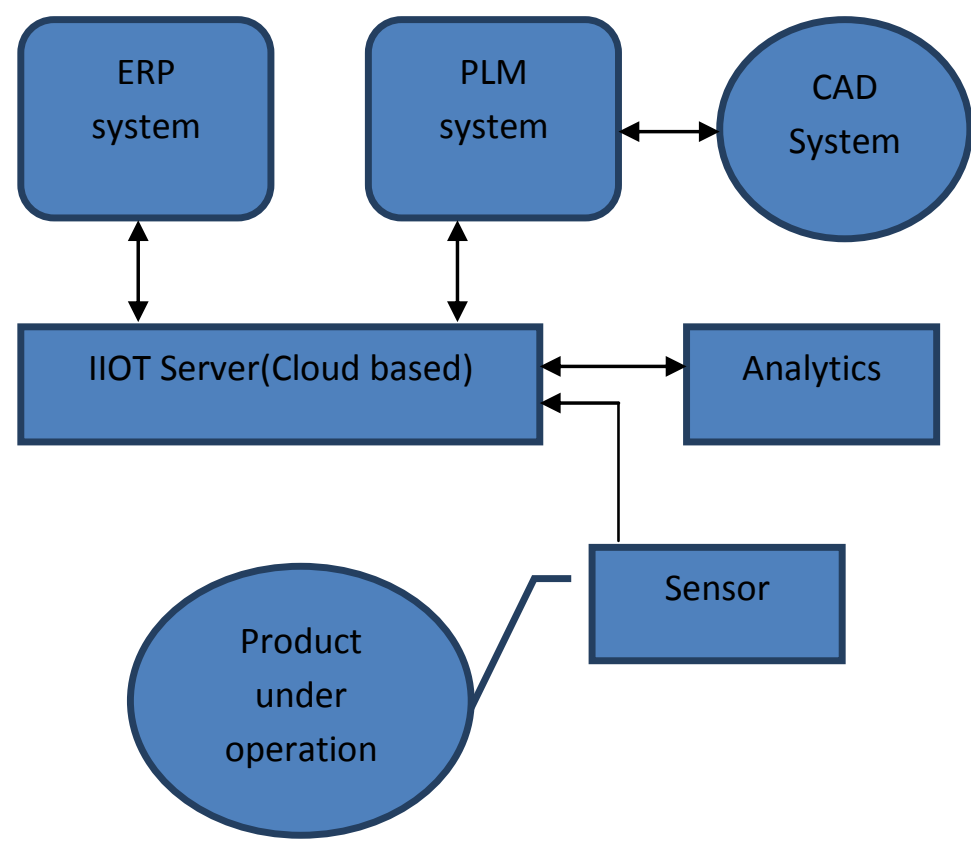

Figure 4 : IOT with PLM and ERP Systems 
So there are various scenarios, the IIoT could be put to use to make the integrations of the ERP and PLM more successful and the best part is that all the software components could be cloud-based to make the whole system cost-effective making it a feasible solution even for (Small and Medium Enterprises) SMEs also. The figure shows a generic representation of such use cases.

\section{CONCLUSION}

With the initiatives like Self-driven cars, Smart Factory system and with concepts like Augmented Reality(AR) and Virtual Reality(VR) among the thick of the things in today's world, its utmost important to have the connected systems working properly. The mission-critical data of a company being managed in PLM and ERP implemented, can be put to enhanced uses with the IoT techniques and IoT enabled equipment. PLM and ERP in the IIOT framework have many use cases defined and put together and put to use by various companies and institutes. The two complimentary components can be made to work to the best potential using the IOT framework. As the number of areas IOT applied to increases so will the maturity of the applications. There are numerous unconventional areas waiting to have the IOT implemented e.g. the room lighting can be IOT controlled and can vary based on the weather of temperature etc.

The application of IoT with PLM and ERP systems could help in planning the spares and maintenance of the production machines or process plant machine, that when broken down could lead to heavy losses.

It could also be used to monitor (in fact remotely!) the condition of railway tracks, telecom towers, airport infrastructure, critical medical equipment, condition of farm in agriculture. There is still scope to utilize the full potential of IoT in various areas in the integrations of ERP and PLM. The applications will be many, depending on the imagination of the designers or product developers.

The area need to be focused upon is, to make the modules of ERP and PLM, IoT enabled and IoT compliant. This will need the strategic will of the EPR and PLM vendors to come together decide the norms and communication protocols keeping the IoT framework in mind. There is a need to have a central authority to formulate these protocols in future to make different products interchangeable since they all will be following the same set of rules and conventions.

\section{REFERENCES}

[1] GREENOUGH, JOHN. "The 'Internet of Things' Will Be The World's Most Massive Device Market And Save Companies Billions Of Dollars."

[2] http://www.businessinsider.in/The-Internet-of-ThingsWill-Be-The-Worlds-Most-Massive-Device-MarketAnd-Save-Companies-Billions-OfDollars/articleshow/44766662.cms

[3] Mahadik, Kiran. 2013. "PLM in the Cloud: Computer System Validation in FDA Regulated Industries." http://www.spkaa.com/tag/product-lifecyclemanagement.

[4] Mckinney, Jim. 2015. "PLM \& ERP: What Is the Difference, and Why Should You Care? Our Mission ..." (January): 1-48.

[5] Mule, Jaykumar Yoga. 2012. "Concept and Evolution of PLM." 4(3): 25-28.

[6] Shilovitsky, Oleg. $2013 . \quad$ "PLM." http://beyondplm.com/2013/04/10/cimdata-plm-forumplm-never-ends/.

[7] Umble, E.J., Haft, R.R. and Umble, M.M., 2003. Enterprise resource planning: Implementation procedures and critical success factors. European journal of operational research, 146(2), pp.241-257.

[8] O'Leary, D.E., 2000. Enterprise resource planning systems: systems, life cycle, electronic commerce, and risk. Cambridge university press.

[9] Schuh, G., Rozenfeld, H., Assmus, D. and Zancul, E., 2008. Process oriented framework to support PLM implementation. Computers in industry, 59(2), pp.210218.

[10] Abramovici, M., 2007. Future trends in product lifecycle management (PLM). In The future of product development (pp. 665-674). Springer Berlin Heidelberg.

[11] Lee, C., Leem, C.S. and Hwang, I., 2011. PDM and ERP integration methodology using digital manufacturing to support global manufacturing. The International Journal of Advanced Manufacturing Technology, 53(1-4), pp.399-409.

[12] Julie Pike, http://www.ptc.com/product-lifecyclereport/complete-product-lifecycle-management-the-iotcomes-to-plm

[13] Jean-François Martin, June 1, 2016 http://mnubo.com/product-lifecycle-managementconnected-object/ 